\title{
Modules that have a supplement in every coatomic extension
}

\author{
Burcu Nişancı Türkmen
}




\title{
MODULES THAT HAVE A SUPPLEMENT IN EVERY COATOMIC EXTENSION
}

\author{
BURCU NIŞANCI TÜRKMEN
}

Received 13 June, 2014

\begin{abstract}
Let $R$ be a ring and $M$ be an $R$-module. $M$ is said to be an $E^{*}$-module (respectively, an $E E^{*}$-module) if $M$ has a supplement (respectively, ample supplements) in every coatomic extension $N$, i.e. $\frac{N}{M}$ is coatomic. We prove that if a module $M$ is an $E E^{*}$-module, every submodule of $M$ is an $E^{*}$-module, and then we show that a ring $R$ is left perfect iff every left $R$-module is an $E^{*}$-module iff every left $R$-module is an $E E^{*}$-module. We also prove that the class of $E^{*}$-modules is closed under extension. In addition, we give a new characterization of left $V$-rings by cofinitely injective modules.
\end{abstract}

2010 Mathematics Subject Classification: 16D50; 16L30

Keywords: supplement, coatomic extension, $E^{*}$-module, $E E^{*}$-module, (semi)perfect ring

\section{INTRODUCTION}

Throughout this paper, we assume that all rings are associative with identity and all modules are unital left modules. Let $M$ be such a module. As usual, the notation $K \subseteq$ $M$ means that $K$ is a submodule of $M$. A submodule $L \subseteq M$ is said to be essential in $M$, denoted as $L \unlhd M$, if $L \cap U \neq 0$ for every non-zero submodule $U \subseteq M$. Dually, a proper submodule $K$ of $M$ is said to be small in $M$ and denoted by $K<<M$, if $M \neq$ $K+T$ for every proper submodule $T$ of $M$. By radical of $M$, denoted by $\operatorname{Rad}(M)$, we will indicate the sum of all small submodules, or, equivalently intersection of all maximal submodules of $M$ (see [8]). If $M=\operatorname{Rad}(M)$, i.e., $M$ has no maximal submodules, $M$ is called radical.

Let $M$ be a module. $M$ is said to be coatomic if $\operatorname{Rad}\left(\frac{M}{K}\right)=\frac{M}{K}$ implies that $K=M$ for some submodule $K$ of $M$ in [9]. $M$ is coatomic if and only if every proper submodule of $M$ is contained in a maximal submodule of $M$. Semisimple modules are coatomic. In addition, every factor module of a coatomic module is again coatomic.

Let $U, V \subseteq M$ be modules. $V$ is called supplement of $U$ in $M$ if it is minimal with respect to $M=U+V$, equivalently $M=U+V$ and $U \cap V<<V$. A submodule $S$ of $M$ has ample supplements in $M$ if, whenever $M=S+L, L$ contains a supplement $K$ of $S$ in $M$ [8]. 
Let $0 \longrightarrow M \longrightarrow N \longrightarrow K \longrightarrow 0$ be a short exact sequence of modules. Then, $N$ is called an extension of $M$ by $K$. To simplify the notation, we think of $M$ as a submodule $N$. In [3], a module $N$ is said to be a cofinite extension of $M$ provided $M \subseteq N$ and $\frac{N}{M}$ is finitely generated. In light of this fact that finitely generated modules are coatomic, we call a module $N$ coatomic extension of $M$ if the factor module $\frac{N}{M}$ of $N$ is coatomic.

It is well known that a module $M$ is injective if and only if it is a direct summand of every extension $N$ of $M$. Since every direct summand is a supplement, Zöschinger studied in [10] modules that have a supplement in every extension and termed these modules the property $(E)$ as a generalization of injective modules. In particular, he proved in [10, Lemma 1.2] that every submodule of a module $M$ has the property $(E)$ if and only if $M$ has ample supplements in every extension, namely the property $(E E)$. It is obvious that the class of modules with the property $(E E)$ contains properly artinian modules.

In [3], a module $M$ is said to have the property $(C E)$ (respectively, the property $(C E E))$ if $M$ has a supplement (respectively, ample supplements) in every cofinite extension. It is shown in [3, Theorem 2.12] that $R$ is semiperfect if and only if every left $R$-module has the property $(C E)$.

Let $M$ be a module. We call $M$ an $E^{*}$-module if $M$ has a supplement in every coatomic extension, and $M$ an $E E^{*}$-module if it has ample supplements in every coatomic extension. The notation of $E^{*}$-modules lies between modules with $(E)$ and modules with the property $(C E)$. Some examples are given to show that these inclusions are proper.

In this paper, we study some basic properties of $E^{*}$-modules and $E E^{*}$-modules. We show that the class of $E^{*}$-modules closed under finite direct sums, extensions and direct summands. We prove that, over a left hereditary ring, every factor module of a coatomic $E^{*}$-module is an $E^{*}$-module. In Proposition 2, we show that if $M$ is an $E E^{*}$-module, then every submodule of $M$ is an $E^{*}$-module. This gives us that over semilocal rings every $E E^{*}$-module is strongly radical supplemented. Also, we prove in Theorem 2 that a ring $R$ is left perfect if and only if every left $R$-module is an $E^{*}$-module if and only if every left $R$-module is an $E E^{*}$-module. In addition, we show that every simple left $R$-module is cofinitely injective if and only if, for every finitely generated left $R$-module $M, \operatorname{Rad}(M)=0$ if and only if $R$ is a left $V$-ring (i.e., rings whose simple left modules are injective). Finally, we prove in Proposition 5 that every $E^{*}$-module over a left $V$-ring is injective.

\section{2. $E^{*}$-Modules ANd $E E^{*}$-Modules}

It is clear that every module with the property $(E)$ is an $E^{*}$-module, but the following example shows that an $E^{*}$-module doesn't have the property $(E)$, in general.

Recall from [1] that a module $M$ is called strongly radical supplemented if every submodule $N$ of $M$ containing $\operatorname{Rad}(M)$ has a supplement in $M$. It is proven in [1, 
Corollary 2.1] that finite sums of strongly radical supplemented modules are strongly radical supplemented. Note that every radical module is strongly radical supplemented.

Example 1. For a non-complete local dedekind domain $R$, let $M$ be the direct sum of left $R$-modules $R^{*}, K^{(I)}$ and $R$, where $R^{*}$ is the completion of $R, K$ is the quotient field of $R$ and $I$ is an index set, respectively. Since injective modules over a dedekind domain are strongly radical supplemented, it follows from [10, Lemma $3.3]$ that $M$ is an $E^{*}$-module. On the other hand, $M$ doesn't have the property $(E)$ by [10, Theorem 3.5].

It is shown in [10, Lemma 1.3 (a)] that direct summands of modules with the property $(E)$ have the property $(E)$. Now we give an analogue of this fact for $E^{*}$ modules.

Proposition 1. Every direct summand of an $E^{*}$-module is an $E^{*}$-module.

Proof. Let $M$ be an $E^{*}$-module and $U$ be a direct summand of $M$. Then, we can write $M=U \oplus V$ for some submodule $V$ of $M$. For any coatomic extension $T$ of $U$, we consider the external direct product of these modules $T$ and $V$. Put $W=T \oplus V$. Now we take the monomorphism $\Phi: M \longrightarrow W$ by $\Phi(m)=\Phi(u+v)=(u, v)$ for all $m=u+v \in U \oplus V=M$. It can be seen that $\Phi(M)$ is an $E^{*}$-module. Now

$$
\frac{W}{\Phi(M)}=\frac{T \oplus V}{\Phi(M)} \cong \frac{T}{U}
$$

is coatomic. It follows that $\Phi(M)$ has a supplement, say $U^{\prime}$, in $W$. Therefore, $T=U+\Psi\left(U^{\prime}\right)$, where $\Psi: W \longrightarrow T$ is the projection. Since $\operatorname{ker}(\Psi) \subseteq \Phi(M)$, we have $U \cap \Psi\left(U^{\prime}\right)<<\Psi\left(U^{\prime}\right)$ by [8, 19.3]. Hence, $\Psi\left(U^{\prime}\right)$ is a supplement of $U$ in $T$.

A submodule of an $E^{*}$-module need not be an $E^{*}$-module, in general. To see this actuality, we shall consider the left $\mathbb{Z}$-modules $\mathbb{Z} \subseteq \mathbb{Q}$. But we have:

Proposition 2. If $M$ is an $E E^{*}$-module, then every submodule $U$ of $M$ is an $E^{*}$-module.

Proof. Let $N$ be a coatomic extension of $U$. We shall show that $U$ has a supplement in $N$. By $W$, we denote the external direct product of $M$ and $N$. Put $F=\frac{W}{W^{\prime}}$, where the submodule $W^{\prime}=\{(u,-u) \in W \mid u \in U\} \subseteq W$. For these inclusion homomorphism $\iota_{1}: U \longrightarrow N$ and $\iota_{2}: U \longrightarrow M$, we can draw the pushout in the following:

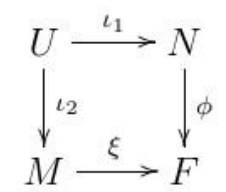


where $\xi$ and $\phi$ are monomorphisms. Then $F=\operatorname{Im}(\xi)+\operatorname{Im}(\phi)$. Therefore $\frac{N}{U} \cong$ $\frac{F}{\operatorname{Im}(\xi)}$ is coatomic. Since $\operatorname{Im}(\xi) \cong M$ is an $E E^{*}$-module, there exists a submodule $L$ of $\operatorname{Im}(\phi)$ such that $L$ is a supplement of $\operatorname{Im}(\xi)$ in $F$. Now

$$
N=\phi^{-1}(F)=\phi^{-1}(\operatorname{Im}(\xi))+\phi^{-1}(L)=U+\phi^{-1}(L)
$$

and

$$
U \cap \phi^{-1}(L)<<\phi^{-1}(L) .
$$

This means that $\phi^{-1}(L)$ is a supplement of $U$ in $N$.

A ring $R$ is called semilocal if $\frac{R}{\operatorname{Rad}(R)}$ is a semisimple artinian ring ([8]). The following corollary is an immediate consequence of Proposition 2.

Corollary 1. Let $M$ be an $E E^{*}$-module over a semilocal ring $R$. Then, $M$ is strongly radical supplemented.

Proof. Let $\operatorname{Rad}(M) \subseteq U \subseteq M$. Then $\frac{M}{U}$ is a factor module of $\frac{M}{\operatorname{Rad}(M)}$. Since $R$ is a semilocal ring, $\frac{M}{\operatorname{Rad}(M)}$ is semisimple as a $\frac{R}{\operatorname{Rad}(R)}$-module. Therefore, $\frac{M}{U}$ is a coatomic $R$-module. By the hypothesis, $U$ has a supplement in $M$. This means that $M$ is strongly radical supplemented.

Let $\Gamma$ be a class of modules. Then, $\Gamma$ is called closed under extension if $M, \frac{N}{M} \in \Gamma$ implies $N \in \Gamma$. The following crucial lemma is used to show that the class of $E^{*}$ modules is closed under extensions.

Lemma 1. Let $M$ be a module and $K$ be a small submodule of $M$. Then, $M$ is coatomic if and only if the factor module $\frac{M}{K}$ is coatomic.

Proof. $(\Longrightarrow)$ It is clear.

$\left(\Longleftarrow\right.$ ) Let $U$ be a proper submodule of $M$. Since $K \ll M$, then $\frac{U+K}{K}$ is a proper submodule of $\frac{M}{K}$. Since $\frac{M}{K}$ is coatomic, $\frac{U+K}{K}$ is contained in a maximal submodule of $\frac{M}{K}$, say $\frac{V}{K}$. Therefore, $V$ is a maximal submodule of $M$. Hence, $M$ is coatomic as required.

Recall from [9, Lemma 1.5 (a)] that the class of coatomic modules is closed under extensions.

Theorem 1. Let $M \subseteq N$ be modules. If $M$ and $\frac{N}{M}$ are $E^{*}$-modules, then $N$ is an $E^{*}$-module.

Proof. Let $K$ be a coatomic extension of $N$. For $M \subseteq N \subseteq K$,

$$
\frac{K}{N} \cong \frac{\frac{K}{M}}{\frac{N}{M}}
$$


is coatomic, and thus $\frac{K}{M}$ is a coatomic extension of $\frac{N}{M}$. By the hypothesis, the submodule $\frac{N}{M}$ has a supplement, say $\frac{L}{M}$, in $\frac{K}{M}$. So we can write $\frac{N}{M}+\frac{L}{M}=\frac{K}{M}$ and $\frac{N}{M} \cap \frac{L}{M}=\frac{N \cap L}{M} \ll \frac{L}{M}$. Therefore, $K=N+L$. Now

$$
\frac{\frac{L}{M}}{\frac{N \cap L}{M}} \cong \frac{L}{N \cap L} \cong \frac{N+L}{N}=\frac{K}{N}
$$

is coatomic. Applying Lemma 1 , we obtain that $\frac{L}{M}$ is coatomic. Since $M$ is an $E^{*}$ module, there exists a submodule $M^{\prime}$ of $L$ such that $M+M^{\prime}=L$ and $M \cap M^{\prime} \ll$ $M^{\prime}$. Then, $K=N+L=N+\left(M+M^{\prime}\right)=N+M^{\prime}$. Assume that $N+M^{\prime \prime}=K$ for some submodule $M^{\prime \prime} \subseteq M^{\prime}$. Then, $M+M^{\prime \prime} \subseteq L$. Since $\frac{L}{M}$ is a supplement of $\frac{N}{M}$ in $\frac{K}{M}$, it follows that $L=M+M^{\prime \prime}$. By the minimality of $M^{\prime}$, we have $M^{\prime \prime}=M^{\prime}$. Therefore $M$ is an $E^{*}$-module.

Note that, by Theorem 1, a finitely generated semisimple module is an $E^{*}$-module.

Corollary 2. Let $M$ be a module and $K$ be a maximal submodule of $M$. If $K$ is an $E^{*}$-module, then $M$ is an $E^{*}$-module. In particular, modules containing a simple maximal submodule are $E^{*}$-modules.

Proof. Let $K$ be an $E^{*}$-module. Since simple modules are $E^{*}$-modules, the factor module $\frac{M}{K}$ is an $E^{*}$-module. Applying Theorem 1, we get $M$ is an $E^{*}$-module.

Now we can prove every finite direct sum of $E^{*}$-modules in the following Proposition.

Proposition 3. Let $M_{i}(i \in I)$ be any finite collection of $E^{*}$-modules and $M=$ $M_{1} \oplus M_{2} \oplus \ldots \oplus M_{n}$. Then, $M$ is an $E^{*}$-module.

Proof. In order to show that $M$ is an $E^{*}$-module, we use induction on $n$. Suppose that $n=2$. Other case can prove by a similar way. Let $M=M_{1} \oplus M_{2}$. Then, $M_{2} \cong$ $\frac{M}{M_{1}}$. By the hypothesis and Theorem 1, we obtain that $M$ is an $E^{*}$-module.

Recall that over a left hereditary ring every factor module of an injective module is injective. In the following, we show that every factor module of a coatomic $E^{*}$ module over a left hereditary ring is an $E^{*}$-module.

Proposition 4. Let $R$ be a left hereditary ring and $M$ be a coatomic $E^{*}$-module. Then every factor module of $M$ is an $E^{*}$-module.

Proof. For any submodule $U$ of $M$, let $N$ be a coatomic extension of $\frac{M}{U}$. Then, $N$ is coatomic. By $E(M)$, we denote the injective hull of $M$. Since $R$ is left hereditary, $\frac{E(M)}{U}$ is injective, and so there exists a commutative diagram with exact rows in the following: 


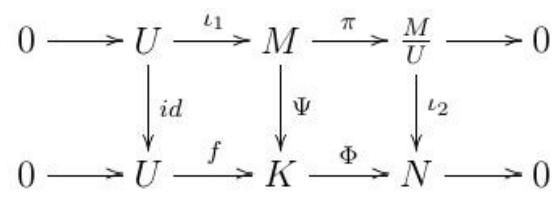

i.e., $f i d=\Psi \iota_{1}$ and $\Phi \Psi=\iota_{2} \pi$, where $\Psi: M \longrightarrow K$ is a monomorphism by [7, Lemma 2.16]. It follows that $N \cong \frac{K}{\Psi(M)}$. Since $M$ is an $E^{*}$-module, $\Psi(M)$ has a supplement, say $V$, in $K$. By the last part proof of Proposition 2, we obtain that $\Phi(V)$ is a supplement of $\frac{M}{U}$ in $N$. Hence, $\frac{M}{U}$ is an $E^{*}$-module.

Recall from [6] that an epimorphism $f: P \longrightarrow M$ is called a small cover if $\operatorname{Ker}(f)<<P$, and a projective module $P$ together with a small cover $f: P \longrightarrow M$ is called a projective cover of $M$. A ring $R$ is called semiperfect if every finitely generated left (or right) $R$-module has a projective cover, and it is called left perfect if every left $R$-module has a projective cover.

It is known that a ring $R$ is semiperfect if and only if $R$ is semilocal and idempotents can be lifted modulo $\operatorname{Rad}(R)$, and it is left perfect if and only if $R$ is semilocal and $\operatorname{Rad}(R)$ is a left t-nilpotent ideal. Local rings are semiperfect ([6]).

Now we give a characterization of left perfect rings via $E^{*}$-modules. Firstly, we have the following lemma.

Lemma 2. The following statements are equivalent over an arbitrary ring.

(1) Every left module is an $E^{*}$-module.

(2) Every left module is an E $E^{*}$-module.

Proof. Suppose that every left module is an $E^{*}$-module. Let $M$ be any module. For a coatomic extension $N$ of $M$, let $N=M+S$ for some submodule $S$ of $N$. Then $\frac{N}{M} \cong \frac{S}{M \cap S}$ is coatomic. By the hypothesis, $M \cap S$ has a supplement in $S$, say $W$. So we can write $S=(M \cap S)+W$ and $(M \cap S) \cap W=M \cap W \ll W$. Then we have $N=M+W$ and $N \cap W \ll W$. Therefore, $M$ is an $E E^{*}$-module. The converse is clear by definitions.

Theorem 2. Let $R$ be a ring. The following three statements are equivalent.

(1) $R$ is left perfect.

(2) Every left $R$-module is an $E^{*}$-module.

(3) Every left $R$-module is an $E E^{*}$-module.

Proof. (1) $\Rightarrow$ (2) By [4, 39.9], over a left perfect ring every left module has the property $(E)$. This completes the proof of (2).

(2) $\Rightarrow$ (3) It follows from Lemma 2.

(3) $\Rightarrow(1)$ Since $E E^{*}$-modules have the property $(C E E)$, by the hypothesis, every $R$-module has the property $(C E E)$. It follows from [3, Theorem 2.12] that $R$ is semiperfect. Therefore, $R$ is semilocal. 
Now it is enough to show that every left $R$-module is strongly radical supplemented by [2, Theorem 1]. Let $M$ be any left $R$-module. By the hypothesis, $M$ is an $E E^{*}$-module. Applying Corollary 1, we deduce that $M$ is strongly radical supplemented.

Now we give an example of a module, which has the property $(C E)$, but not an $E^{*}$-module.

Example 2. Let $p$ be a prime integer in $\mathbb{Z}$. Consider the local dedekind domain $R=\mathbb{Z}_{(p)}=\left\{\frac{a}{b} \mid a, b \in \mathbb{Z}\right.$ and $\left.p \nmid b\right\}$. Let $N$ be the left $R$-module $R^{(\mathbb{N})}$. Put $M=\operatorname{Rad}(N)$. Since $R$ is a local ring, the factor module $\frac{N}{M}$ is semisimple as a $\frac{R}{\operatorname{Rad}(R)}-$ module. Therefore, $\frac{N}{M}$ is a semisimple $R$-module and so $N$ is a coatomic extension of $M$. It follows from [3, Theorem 2.12] that $M$ has the property $(C E)$. On the other hand, $M$ is not an $E^{*}$-module by [2, Theorem 1].

In [5], a ring $R$ is said to be a left $V$-ring if every simple left $R$-module is injective. It is well known that a ring $R$ is a left $V$-ring if and only if $\operatorname{Rad}(M)=0$ for every left $R$-module $M$. Recall from [3] that a module $M$ is called cofinitely injective if $M$ is a direct summand of every cofinite extension $N$ of $M$. Clearly, injective modules are cofinitely injective, and a cofinitely injective module has the property $(C E)$. Now we have the next result:

Proposition 5. Let $R$ be a left $V$-ring and $M$ be an $E^{*}$-module over the ring. Then, $M$ is injective.

Proof. Let $N$ be an extension of $M$. Since every module over a left $V$-ring is coatomic, $\frac{N}{M}$ is coatomic. Therefore, $N$ is a coatomic extension of $M$. By assumption, we can write $N=M+K$ and $M \cap K<<K$ for some submodule $K \subseteq N$. Since $R$ is a left $V$-ring, we obtain that $M \cap K \subseteq \operatorname{Rad}(K) \subseteq \operatorname{Rad}(N)=0$. This means that $M$ is a direct summand of $N$. Hence, $M$ is injective.

Theorem 3. The following statements are equivalent for a ring $R$.

(1) Every simple left $R$-module is cofinitely injective.

(2) If $M$ is a finitely generated left $R$-module, $\operatorname{Rad}(M)=0$.

(3) Every proper left ideal I of $R$ is an intersection of maximal left ideals.

Proof. (1) $\Longrightarrow$ (2) Let $M$ be an arbitrary finitely generated left $R$-module and let $m \in \operatorname{Rad}(M)$. We claim that $m=0$. Put $K=R m$. Then, $K$ has a maximal submodule $L$. Therefore, $\frac{K}{L}$ is a simple left $R$-module and by assumption $\frac{K}{L}$ is cofinitely injective. Now, for $L \subseteq K \subseteq M$ modules,

$$
\frac{\frac{M}{L}}{\frac{K}{L}} \cong \frac{M}{K}
$$


is coatomic since $M$ is finitely generated. So there exists the decomposition $\frac{M}{L}=$ $\frac{K}{L} \oplus \frac{T}{L}$ for some submodule $\frac{T}{L} \subseteq \frac{M}{L}$. Note that

$$
\frac{K}{L} \cong \frac{\frac{M}{L}}{\frac{T}{L}} \cong \frac{M}{T}
$$

is simple. Thus, $T$ is a maximal submodule of $M$. Therefore, $m \in K \cap T \subseteq L$. It follows that $m=0$.

(2) $\Longrightarrow$ (3) and (3) $\Longrightarrow$ (1) follow from [5, Theorem 6.1].

As a consequence of the above, we have the following.

Corollary 3. Let $R$ be a ring. Then, $R$ is a left $V$-ring if and only if every simple left $R$-module is cofinitely injective.

Proof. The proof follows from Theorem 3 and [5, Theorem 6.1].

\section{ACKNOWLEDGEMENT}

I would like to thank the referee for the valuable suggestions and comments which improved the revision of the paper.

\section{REFERENCES}

[1] E. Büyükaşık and E. Türkmen, "Strongly radical supplemented modules," Ukrainian Math. J., vol. 63, no. 8, pp. 1306-1313, 2012. [Online]. Available: http://dx.doi.org/10.1007/ s11253-012-0579-3

[2] E. Büyükaşik and C. Lomp, "Rings whose modules are weakly supplemented are perfect. Applications to certain ring extensions," Math. Scand., vol. 105, no. 1, pp. 25-30, 2009.

[3] H. Çalışıcı and E. Türkmen, "Modules that have a supplement in every cofinite extension," Georgian Math. J., vol. 19, no. 2, pp. 209-216, 2012. [Online]. Available: http://dx.doi.org/10.1515/gmj-2012-0018

[4] J. Clark, C. Lomp, N. Vanaja, and R. Wisbauer, Lifting modules, ser. Frontiers in Mathematics. Birkhäuser Verlag, Basel, 2006, supplements and projectivity in module theory.

[5] S. K. Jain, A. K. Srivastava, and A. A. Tuganbaev, Cyclic modules and the structure of rings, ser. Oxford Mathematical Monographs. Oxford University Press, Oxford, 2012. [Online]. Available: http://dx.doi.org/10.1093/acprof:oso/9780199664511.001.0001

[6] F. Kasch, Modules and rings, ser. London Mathematical Society Monographs. Academic Press, Inc. [Harcourt Brace Jovanovich, Publishers], London-New York, 1982, vol. 17, translated from the German and with a preface by D. A. R. Wallace.

[7] S. Özdemir, "Rad-supplementing modules," ArXiv e-prints, Oct. 2012.

[8] R. Wisbauer, Foundations of module and ring theory, ser. Algebra, Logic and Applications. Gordon and Breach Science Publishers, Philadelphia, PA, 1991, vol. 3, a handbook for study and research.

[9] H. Zöschinger, "Komplementierte Moduln über Dedekindringen,” J. Algebra, vol. 29, pp. 42-56, 1974.

[10] H. Zöschinger, "Moduln, die in jeder Erweiterung ein Komplement haben," Math. Scand., vol. 35, pp. 267-287, 1974. 
Author's address

\section{Burcu Nişancı Türkmen}

Amasya University, Department of Mathematics, İpekkoy, 05100 Amasya, Turkey

E-mail address: burcunisancie@hotmail.com 